\title{
Quiet eye training facilitates competitive putting performance in elite golfers
}

\section{Samuel J. Vine, Lee J. Moore and Mark R. Wilson*}

Sport and Health Sciences, University of Exeter, Exeter, UK

\section{Edited by:}

Jared Porter, Southern Illinois

University, USA

Reviewed by:

Bettina Edna Blaesing, Bielefeld University, Germany

Joan Vickers, University of Calgary,

Canada

${ }^{*}$ Correspondence:

Mark R. Wilson, Sport and Health

Sciences, University of Exeter, St.

Luke's Campus, Exeter EX1 2LU, UK.

e-mail:mark.wilson@ex.ac.uk
The aim of this study was to examine the effectiveness of a brief quiet eye (QE) training intervention aimed at optimizing visuomotor control and putting performance of elite golfers under pressure, and in real competition. Twenty-two elite golfers (mean handicap 2.7) recorded putting statistics over 10 rounds of competitive golf before attending training individually. Having been randomly assigned to either a QE training or Control group, participants were fitted with an Applied Science Laboratories Mobile Eye tracker and performed 20 baseline (pre-test) putts from $10 \mathrm{ft}$. Training consisted of video feedback of their gaze behavior while they completed 20 putts; however the QE-trained group received additional instructions related to maintaining a longer QE period. Participants then recorded their putting statistics over a further 10 competitive rounds and re-visited the laboratory for retention and pressure tests of their visuomotor control and putting performance. Overall, the results were supportive of the efficacy of the QE training intervention. QE duration predicted $43 \%$ of the variance in putting performance, underlying its critical role in the visuomotor control of putting. The QE-trained group maintained their optimal QE under pressure conditions, whereas the Control group experienced reductions in $\mathrm{QE}$ when anxious, with subsequent effects on performance. Although their performance was similar in the pre-test, the QE-trained group holed more putts and left the ball closer to the hole on missed putts than their Control group counterparts in the pressure test. Importantly, these advantages transferred to the golf course, where QE-trained golfers made 1.9 fewer putts per round, compared to pre-training, whereas the Control group showed no change in their putting statistics. These results reveal that QE training, incorporated into a pre-shot routine, is an effective intervention to help golfers maintain control when anxious.

Keywords: golf putting, gaze, anxiety, quiet eye, visuomotor control

\section{INTRODUCTION}

Given its relative importance within the game of golf it is unsurprising that much research has focused on improving accuracy in golf putting (Hellstrom, 2009a). Requiring precise and accurate movements and often performed under conditions of high pressure, the golf putt is a skill particularly susceptible to breakdown (Gucciardi et al., 2010). A major focus for research examining the acquisition and performance of far-aiming skills, like putting, has been the control of gaze. Research has revealed proficiency related differences in gaze control, with experts demonstrating more efficient gaze strategies across a range of sport skills (see Mann et al., 2007 for a review). Gaze control has also been shown to be susceptible to change under heightened levels of anxiety, with less efficient gaze leading to reductions in performance (see Janelle, 2002; Wilson, 2008 for reviews). Researchers have therefore suggested that training interventions, designed to guide optimal gaze control, may facilitate performance in highly pressurized environments (Vickers and Williams, 2007; Behan and Wilson, 2008). The current study aimed to assess the utility of a gaze training intervention aimed at improving golf putting among low handicap golfers.

The control of gaze has been shown to be an important determinant of accuracy in the execution of motor tasks (Land, 2009; Vickers, 2009). Vickers (1992) was the first to assess the gaze control of expert and novice golfers whilst performing golf putts. Vickers highlighted proficiency differences in gaze control during the alignment and execution phase of the putt. Most notably, experts kept their eyes steady on the back of the ball for around two seconds prior to the initiation of the back swing and maintained this fixation until the putter contacted the ball. Their gaze would then remain steady in the same location for around 300-500 ms after the ball had been struck. This aiming fixation was later termed the quiet eye (QE; Vickers, 1996). The QE has since been shown to underlie higher levels of skill and performance in a wide range of aiming and interceptive skills; with experts having longer QE durations than non-experts and successful attempts having longer QE durations than unsuccessful attempts (see Mann et al., 2007; Vickers, 2009 for reviews).

Vickers proposed that the $\mathrm{QE}$ is a period of time when task relevant environmental cues are processed and motor plans are coordinated for the successful completion of the upcoming task. This explanation of how the QE may "work" resonates with the attentional systems outlined by Corbetta and colleagues (Corbetta and Shulman, 2002; Corbetta et al., 2008). The top-down, goaldirected attentional system (dorsal attention), is important for response or action selection and is involved in linking relevant stimuli to motor planning. The stimulus-driven attentional system (ventral attention) works as a circuit breaker for the dorsal system, directing attention to salient cues. Theoretically, longer QE periods 
therefore allow performers an extended duration of programming (dorsal attention), while minimizing distraction (ventral attention) from other internal or environmental cues.

From an applied perspective, such insights into the psychomotor processes underpinning skilled performance may guide the development of innovative training interventions to optimize gaze control and facilitate accurate and efficient motor task performance. Research by Vickers and colleagues has shown that skilled performers can be taught to develop longer and more effective QE periods, with subsequent improvements in performance (Adolphe et al., 1997; Harle and Vickers, 2001). For example, Harle and Vickers (2001) examined the effect of a QE training regime aimed at improving the gaze control and free-throw accuracy of near-elite basketball players. Results showed that not only did the QE-trained team significantly increase their QE durations from an average of 300-900 ms and free-throw percentages by $12 \%$ in a laboratory setting, but they improved their competitive free-throw percentage by $22.6 \%$ after two seasons.

Research examining disruptions in gaze control during pressurized performance may provide additional information as to how training interventions can facilitate performance (Wilson, 2008). Effective attentional control, as indexed by the QE, is negatively impacted by anxiety, causing subsequent performance degradation in far-aiming tasks (Vickers and Williams, 2007; Behan and Wilson, 2008; Wilson et al., 2009; Vine and Wilson, 2010; Wood and Wilson, 2010). However, Vickers and Williams (2007) found that elite biathletes who managed to maintain their QE durations under pressure were less susceptible to choking. The authors suggested that by allocating attention externally to critical task information (via the QE) the biathletes were insulated from the normally debilitating effects of anxiety.

This finding raises fascinating questions about whether performers can be taught to maintain effective attentional control under pressure, via QE training (Wilson et al., 2010b). In line with this notion, a recent study examined the effects of a QE training regime aimed at optimizing the gaze control, learning, and performance under pressure of novice golfers in a putting task (Vine and Wilson, 2010). Consistent with the work of Vickers and colleagues, the results revealed that the QE-trained golfers displayed significantly longer and more effective QE durations, as well as more accurate putting performance post-training when compared to pre-training. Furthermore, the authors found that the elevated anxiety encountered in a subsequent pressure test had a different effect on the gaze control and performance of QE-trained and Control groups. Specifically, the Control group displayed significantly shorter QE durations and performed significantly worse in a pressure test than their QE-trained counterparts. Thus, the results indicate that QE training acted to protect performers from the adverse effects of anxiety upon performance by maintaining effective QE durations.

\section{AIMS AND HYPOTHESES}

The aims of this research were to extend the findings of Vine and Wilson (2010) using a brief QE intervention aimed at training optimal visuomotor control of elite golfers in a laboratory setting and under conditions of heightened anxiety (pressure test). Furthermore, the research aimed to examine whether the
QE training would result in improvements in real competitive putting performance. In line with previous research it was predicted that participants in a QE-trained group would perform significantly better in laboratory-based retention and pressure tests, compared to those in a Control group, due to more optimal gaze control (QE). Furthermore, this in turn, was expected to contribute to participants in the QE-trained group performing significantly better in real competitive settings, compared to those in a Control group. Specifically, it was hypothesized that:

1. The QE-trained group would demonstrate significantly better performance and gaze control (longer QE durations) in a laboratory-based retention test, compared to their Control group counterparts.

2. The QE-trained group would demonstrate significantly better performance and gaze control (longer QE durations) in a laboratory-based pressure test, compared to their Control group counterparts.

3. The QE-trained group would demonstrate significantly greater improvements in competitive performance pre- to posttraining, compared to the Control group.

\section{MATERIALS AND METHODS PARTICIPANTS}

Twenty-two elite male golfers (mean age $20.95, \mathrm{SD}=2.66$ ) with an average handicap of $2.78(\mathrm{SD}=2.24)$ volunteered to take part in the study. All participants currently held a handicap less than 6 and thus, were identified as elite (as Karlsen et al., 2008). All handicaps were obtained via self-report and confirmed by each golfer's respective club secretary. All were right handed, reported normal or corrected vision and were individually tested. Local ethics committee approval was obtained prior to the start of testing and participants provided written informed consent.

\section{APPARATUS}

Straight putts were taken from 3, $10 \mathrm{ft}(3.05 \mathrm{~m})$ locations to a regulation hole $(10.80 \mathrm{~cm}$ diameter $)$ on an artificial putting green (length $=6 \mathrm{~m}$, width $=2.5 \mathrm{~m}$ ). All participants used their own golf putters and standard size $(4.27 \mathrm{~cm}$ diameter) white golf balls. Consistent with recent QE research (Behan and Wilson, 2008; Wilson and Pearcey, 2009; Wilson et al., 2009; Vine and Wilson, 2010) gaze was measured using an Applied Science Laboratories (ASL; Bedford, MA, USA) Mobile Eye Tracker. This lightweight system uses two features; the pupil and corneal reflection (determined by the reflection of an infrared light source from the surface of the cornea) to calculate point of gaze (at $29 \mathrm{~Hz}$ ) relative to eye and scene cameras mounted on a pair of spectacles. A laptop (Lenovo R500 ThinkPad) installed with "Eyevision" (ASL) recording software was incorporated with the system. A circular cursor, representing $1^{\circ}$ of visual angle with a $4.5-\mathrm{mm}$ lens, indicating the location of gaze in a video image of the scene (spatial accuracy of $\pm 0.5^{\circ}$ visual angle; $0.1^{\circ}$ precision) is viewed in real time on the laptop and recorded for offline analysis. The experimenter and laptop were positioned behind and to the left of the participant to minimize distraction. 


\section{MEASURES}

\section{Experimental performance}

Two measures of experimental putting performance were calculated; performance outcome (percentage of putts holed) and performance error (the distance that the ball finished from the hole, in $\mathrm{cm}$ ). Zero was recorded and used in the calculation of average performance error on trials where the putt was holed. These measures are befitting with those utilized in previous golf putting research (e.g., Wilson et al., 2007; Wilson and Pearcey, 2009; Cooke et al., 2010).

\section{Competitive performance}

Two measures of competitive putting performance were derived from putting statistics kept by the participants over 20 competitive rounds (before and after the laboratory-based training session). Participants recorded the number of putts taken per hole, whether they had a putt at a distance of 6-10 ft on each hole and if they were successful with that putt on a putting scorecard. These data were used to calculate measures including average number of putts per round and the percentage of putts holed from 6 to $10 \mathrm{ft}$. A low number of putts per round and a high percentage of putts holed from 6 to $10 \mathrm{ft}$ represented better performance (see Pelz, 2000).

\section{State anxiety}

Cognitive state anxiety was measured throughout the testing period using the Mental Readiness Form 3 (MRF-3; Krane, 1994). The MRF-3 has three, bipolar, 11 point Likert scales that are anchored between worried-not worried for the cognitive anxiety scale, tensenot tense for the somatic anxiety scale, and confident-not confident for the self-confidence scale. The MRF-3 is a shorter and more expedient alternative to the Competitive State Anxiety Inventory 2 (CSAI-2; Martens et al., 1990), allowing anxiety to be reported during, as well as prior to performance. Krane's validation work revealed correlations between the MRF-3 and the CSAI- 2 subscales of 0.76 for cognitive anxiety, 0.69 for somatic anxiety, and 0.68 for self-confidence. The MRF-3 has also been used in previous research examining the impact of cognitive anxiety in golf putting (e.g., Wilson et al., 2007; Vine and Wilson, 2010).

\section{Quiet eye duration}

The QE duration was operationally defined as the final fixation toward the ball prior to the initiation of the backswing (as Vickers, 1992, 2007; Wilson and Pearcey, 2009; Vine and Wilson, 2010). A fixation was defined as a gaze maintained on a location within $1^{\circ}$ of visual angle for a minimum of $120 \mathrm{~ms}$ (Wilson et al., 2009). QE onset occurred before the putter started the backswing movement away from the ball and QE offset eventuated when the gaze deviated off the fixated location by $1^{\circ}$ or more, for greater than $120 \mathrm{~ms}$. If the cursor disappeared for one or two frames (e.g., a blink) and then returned to the same location, the QE duration resumed. QE durations were calculated using Quiet Eye Solutions software (QE solutions Inc.).

\section{PROCEDURE}

After reading the written information introducing the study and being informed of their right to withdraw from the study at any time, participants provided informed consent. Next, participants were provided with putting scorecards and recorded their competitive putting performance for 10 competition rounds (over a maximum of 3 months). This data acted as a baseline (pre-training) measure. Upon completion of these 10 rounds participants attended the laboratory individually for training and the first testing session.

On arrival participants were randomly assigned to either a QE training or Control group. Next, participants were given the chance to take 10 practice putts and familiarize themselves with the surroundings. Participants were then fitted with the eye tracker and calibration took place. During calibration participants adopted their putting stance and were then asked to fixate in turn on one of four golf balls placed in a square on the green approximately half a meter in front of their feet. Calibration was repeated every 10 putts to ensure the eye tracker had not been knocked or altered, and if necessary the line of gaze was re-calibrated before proceeding with the testing protocol.

Following calibration, participants took a further 10 practice putts to become familiar with putting whilst wearing the eye tracker. Next, participants took 20 putts (2 blocks of 10), during which experimental performance and gaze behavior $(\mathrm{QE})$ were recorded. This data acted as a baseline (pre-test) measure. Participants then began their assigned training regime (QE or Control; see Training Protocol). In both training regimes participants took a further 20 putts (four blocks of five putts), during which experimental performance and gaze behavior were measured. Following cessation of the training regimes participants were provided with more putting scorecards and dates were arranged for them to return to the laboratory.

After recording their putting performance for another 10 competitive rounds (again over a maximum of 3 months), participants returned to the laboratory for a second testing session. Familiarization and calibration were repeated in the same manner as during their first visit. Following calibration, participants completed 20 putts in a retention task and a further 15 putts in a pressure test (high anxiety; see Pressure Manipulation). Anxiety levels (MRF-3), experimental performance, and gaze behavior were recorded throughout these series of putts. Finally, participants were thanked and debriefed about the aims of the study, and offered feedback if requested.

\section{TRAINING PROTOCOL}

The training regimes were adapted from previous QE training research (Harle and Vickers, 2001; Vickers, 2007; Vine and Wilson, 2010). Both training groups started by viewing their own gaze data from putts taken during the pre-test and were asked to verbalize what they noticed with regard to their gaze control. Next, after taking five putts, during which performance and gaze control measures were recorded, both groups viewed a video of their gaze control alongside that of an elite prototype who exhibited the critical QE as found in past gaze research (Vickers, 1992, 2007). At this stage only the QE-trained group were directed by the researcher toward key differences between their gaze control and that of the elite prototype. The researcher asked questions to elicit the QE-trained participants' understanding of their gaze control relative to the expert. Furthermore, consistent with previous QE training research (e.g., Harle and Vickers, 2001; Vine and Wilson, 2010), the following points adapted from Vickers (2007) were stressed: 
1. Assume your stance and align the club so the gaze is on the back of the ball.

2. After setting up over the ball, fix your gaze on the hole. Fixations toward the hole should be made no more than 3 times.

3. The final fixation should be a QE on the back of the ball. The onset of the QE should occur before the stroke begins and last for 2 to 3 seconds.

4. No gaze should be directed to the clubhead during the backswing or foreswing.

5. The QE should remain on the green for 200 to $300 \mathrm{~ms}$ after the club contacts the ball.

Next, the QE-trained group were asked to improve aspects of their gaze control based on these feedback points and what they had learned from viewing the elite prototype. Both groups then performed a further 15 putts, during which performance and gaze control measures were recorded.

\section{PRESSURE MANIPULATION}

Consistent with previous research several techniques were used to create high levels of cognitive anxiety for the pressure test (see Wilson et al., 2009; Vine and Wilson, 2010). Firstly, a competition was set up whereby participants were informed that the individual

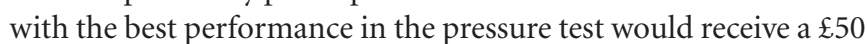
cash reward. Secondly, participants were told their scores would be compared with others taking part and may be sent to and used by their respective golf courses. Non-contingent feedback was also employed, with participants informed that their previous 20 putts (retention test) would put them in the bottom $30 \%$ when compared to those that had already taken part in the competition. They were encouraged to try and improve upon their performance otherwise their data would be of no use for the study.

\section{DATA ANALYSIS}

Video data from the Mobile Eye were analyzed after the testing period using Quiet Eye Solutions software (Quiet Eye Solutions Inc.). This software allows for detailed frame-by-frame coding of both the motor action (recorded from the Mobile Eye's scene camera at $29 \mathrm{~Hz}$ ) and the gaze behavior of the performer, creating "visionin-action" data (Vickers, 2007). Once the gaze and motor phases have been coded, the software automatically calculates the QE with respect to the initiation of the backswing of the putter. Congruent with previous QE research (e.g., Behan and Wilson, 2008; Wilson et al., 2009; Vine and Wilson, 2010), a subset of putts (every fourth) were selected for analysis. However, due to a digital storage device failure video data from the eye tracker for five of the QE-trained participants was lost, and subsequently could not be analyzed.

\section{STATISTICAL ANALYSIS}

Test phase (retention test and pressure test) anxiety data were subjected to a 2 (Group: QE-trained, Control) $\times 2$ (Test: retention, pressure) mixed design analysis of variance (ANOVA). Pre-test and test phase performance and QE data were subjected to a 2 (Group: QE-trained, Control) $\times 3$ (Test: pre-test, retention, pressure) mixed design ANOVA. Competitive performance measures were subjected to a 2 (Group: QE-trained, Control) $\times 2$ (Time: pre-training, post-training) mixed design ANOVA. Significant main and interaction effects were followed up with Bonferroni corrected post hoc $t$-tests and simple $t$-tests, respectively. Where the sphericity assumption was violated, Greenhouse-Geisser corrections were applied. Effect sizes were calculated using partial eta squared $\left(\eta_{\mathrm{p}}^{2}\right)$ for omnibus comparisons. Linear regression analysis was also performed on the $\mathrm{QE}$ and performance error data for the test phase to assess the degree to which QE durations predicted variance in performance.

\section{RESULTS \\ ANXIETY}

Analysis of variance revealed a significant main effect for test $\left[F(1,20)=265.55, p<0.001, \eta_{\mathrm{p}}^{2}=0.93\right]$, with anxiety being significantly higher during the pressure test than the retention test $[t(21)=-16.75, p<0.001]$. There was no significant main effect for group $\left[F(1,20)=1.29, p=0.27, \eta_{\mathrm{p}}^{2}=0.06\right]$, and no significant interaction effect $\left[F(1,20)=0.13, p=0.73, \eta_{\mathrm{p}}^{2}=0.01\right]$, indicating that both groups reported comparable levels of anxiety. While cognitive anxiety was the main focus of analysis, the self-report data from all three MRF-3 scales are presented in Table 1.

\section{QUIET EYE}

Analysis of variance revealed a significant main effect for test $\left[F(2,30)=3.50, p<0.05, \eta_{p}^{2}=0.19\right]$, and group $[F(1,15)=9.48$, $\left.p<0.01, \eta_{\mathrm{p}}^{2}=0.39\right]$. This was accompanied by a significant interaction effect $\left[F(2,30)=5.82, p<0.01, \eta_{p}^{2}=0.28\right]$. Follow-up $t$-tests revealed no significant $\mathrm{QE}$ differences between groups for pre-test $[t(15)=-0.40, p=0.70]$, or retention test $[t(15)=1.84, p=0.09]$. However, the QE-trained group $($ mean $=2794.31, \mathrm{SD}=1136.11)$ did display significantly longer QE durations than the Control group $($ mean $=1404.74, \mathrm{SD}=489.48)$ during the pressure test $[t(7.58)=3.04, p<0.05]$. Pre-test and test phase QE data are presented in Figure 1.

\section{PERCENTAGE PUTTS HOLED (LABORATORY)}

Analysis of variance revealed no significant main effect for test $\left[F(1.37,27.43)=0.55, p=0.52, \eta_{\mathrm{p}}^{2}=0.03\right]$. However, there was a significant main effect for group $[F(1,20)=8.64$, $\left.p<0.01, \eta_{\mathrm{p}}^{2}=0.30\right]$, and a significant interaction effect $\left[F(1.37,27.43)=5.97, p<0.05, \eta_{p}^{2}=0.23\right]$. Follow-up $t$-tests showed that there were no significant differences in putts holed between groups for pre-test [ $t(20)=0.14, p=0.89$ ], or retention test $[t(20)=2.35, p=0.087]$. However the QE-trained group (mean $=60 \%, \mathrm{SD}=15 \%)$ did hole a significantly higher percentage of putts than the Control group ( mean $=36 \%, \mathrm{SD}=15 \%)$ during the pressure test $[t(20)=3.75, p<0.005]$. Pre-test and test phase percentage holed data are also presented in Figure 1.

Table 1 | Mean (standard deviation) scores from MRF-3 questionnaire for QE-trained and Control groups across the test phase.

\begin{tabular}{lllllll}
\hline & \multicolumn{2}{c}{ Retention test } & & \multicolumn{2}{c}{ Pressure test } \\
\cline { 2 - 3 } \cline { 5 - 6 } & QE-trained & Control & & QE-trained & Control \\
\hline Cognitive anxiety & $3.04(0.94)$ & $3.40(0.88)$ & & $7.17(0.54)$ & & $7.35(0.82)$ \\
Somatic anxiety & $3.04(0.50)$ & $3.40(1.35)$ & & $6.88(0.77)$ & $7.00(0.75)$ \\
Self-confidence & $9.00(0.93)$ & $7.75(1.77)$ & & $4.92(1.22)$ & $3.20(0.89)$
\end{tabular}




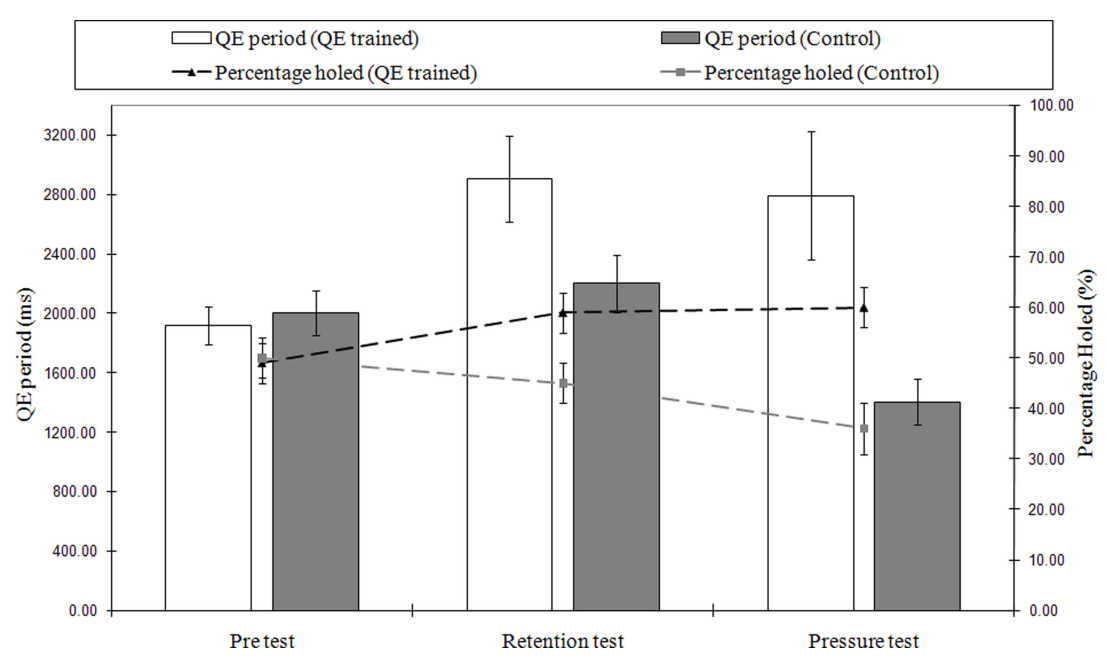

FIGURE 1 | Quiet eye period (ms) and percentage of putts holed (\%) for QE-trained and Control groups across pre-test, retention test, and pressure test conditions ( \pm SEM).

\section{AVERAGE PERFORMANCE ERROR (LABORATORY)}

Analysis of variance revealed no significant main effect for test $\left[F(2,40)=1.97, p=0.15, \eta_{\mathrm{p}}^{2}=0.09\right]$. However, there was a significant main effect for group $\left[F(1,20)=21.58, p<0.001, \eta_{\mathrm{p}}^{2}=0.052\right]$, and a significant interaction effect $\left[F(2,40)=5.77, p<0.01, \eta_{\mathrm{p}}^{2}=0.22\right]$. Follow-up $t$-tests revealed no significant differences between groups for pre-test $[t(14.33)=0.13, p=0.90]$. However, the QE-trained group (mean $=4.58, \mathrm{SD}=2.98$ ) did perform significantly better than the Control group ( mean $=8.37, \mathrm{SD}=3.51$ ) during the retention test $[t(20)=2.74, p<0.05]$. Furthermore, the QE-trained group (mean $=4.45, \mathrm{SD}=1.04$ ) performed significantly better than the Control group $($ mean $=10.28, \mathrm{SD}=4.49)$ during the pressure test $[t(9.81)=4.01, p<0.005]$. Pre-test and test phase average performance error data are presented in Figure 2.

\section{REGRESSION ANALYSIS}

Results from the regression analysis revealed that QE duration predicted $43 \%$ of the variance in average performance error during the test phase $\left(R^{2}=0.43, \beta=13.93, p<0.005\right)$.

\section{COMPETITIVE PERFORMANCE \\ Putts per round}

Analysis of variance revealed no significant main effect for group $\left[F(1,20)=2.20, p=0.15, \eta_{p}^{2}=0.10\right]$. However, there was a significant main effect for time $\left[F(1,20)=13.92, p<0.005, \eta_{p}^{2}=0.41\right]$, and a significant interaction effect $\left[F(1,20)=11.70, p<0.005, \eta_{p}^{2}=0.37\right]$. Follow-up $t$-tests revealed no significant differences between groups at pre-training $[t(20)=0.55, p=0.59]$, however, the QE-trained group $($ mean $=27.61, \mathrm{SD}=1.93)$ did display significantly fewer putts per round than the Control group (mean $=29.89, \mathrm{SD}=3.11)$ at post-training $[t(20)=2.11, p<0.05]$. Within groups, the QE-trained group displayed a significant reduction in putts per round from pre-training to post-training $[t(11)=6.18, p<0.001]$, whilst the Control group displayed no significant difference in performance between pre-training and post-training $[t(9)=0.18, p=0.86]$. Putts per round data are presented in Figure 3.

\section{Percentage 6-10 ft putts holed}

Analysis of variance revealed no significant main effect for group $\left[F(1,20)=1.75, p=0.20, \eta_{\mathrm{p}}^{2}=0.08\right]$, and no significant interaction effect $\left[F(1,20)=2.33, p=0.14, \eta_{\mathrm{p}}^{2}=0.10\right]$. However, there was a significant main effect for time $\left[F(1,20)=5.36, p<0.05, \eta_{p}^{2}=0.21\right]$. Follow-up tests revealed no significant difference between groups at pre-training $(p=0.686)$ however the QE-trained group holed significantly more putts from 6 to $10 \mathrm{ft}$ than the Control group post-training $(p<0.05)$. The percentage of holed putts data are presented in Figure 3.

\section{DISCUSSION}

The purpose of the present study was to examine the effectiveness of a brief QE training intervention aimed at optimizing the gaze control and putting performance of elite golfers in a laboratory setting and under conditions of heightened anxiety. Furthermore, the present study aimed to test whether the potential benefits of training the QE translated into improvements in real competitive putting performance.

\section{HYPOTHESIS 1: RETENTION}

There were no differences in the performance levels (percentage holed or average performance error) of both groups at baseline (pre-test), indicating that both groups started from similar levels of performance. Contrary to the predictions of hypothesis 1, although the QE-trained group holed more putts than their Control group counterparts during the retention test ( 45 vs. $59 \%$ ) this difference was not statistically significant (Figure 1). However, the QE-trained group did exhibit significantly lower average performance error than the Control group (4.58 vs. $8.37 \mathrm{~cm}$; Figure 2), reflecting that they consistently left the ball closer to the hole on misses, demonstrating superior distance control; a strong determinant of putting proficiency (Tierney and Coop, 1998).

The QE data (Figure 1) revealed that both groups had comparable QE durations at baseline (pre-test), suggesting that any subsequent changes in duration should be as a result of the intervention. As 


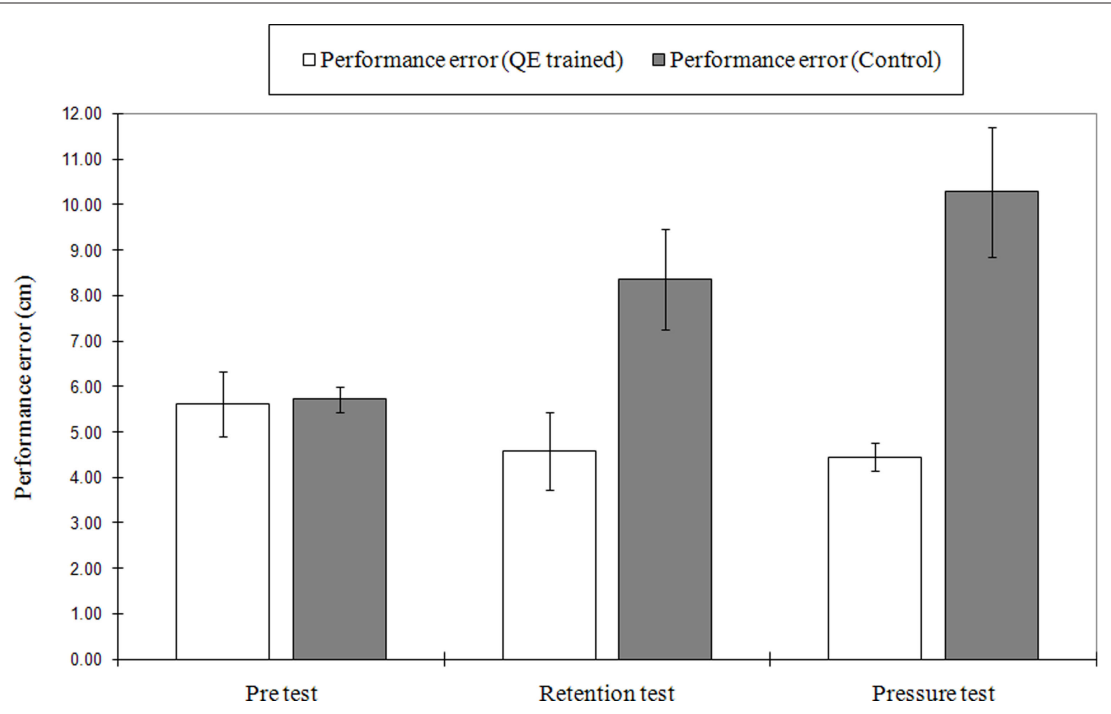

FIGURE 2 | Putting performance error (cm) for QE-trained and Control groups across pre-test, retention test, and pressure test conditions ( \pm SEM).

expected, while the Control group displayed no significant change in QE from pre-test to retention test $(p=0.31)$, the QE-trained group displayed a significant increase $(p<0.05)$ in QE duration, supporting the effectiveness of the training intervention. However, in contrast to the predictions of hypothesis 1, although the QE-trained group displayed longer mean QE durations than the Control group ( 2817 vs. $2203 \mathrm{~ms}$ ) during the retention test, this difference was not statistically significant (Figure 1).

Although differences in the percentage of putts holed and QE duration in the retention test were non-significant, they were in the predicted direction. The regression analysis adds further support to the importance of longer QE periods in putting, with longer QE periods being strongly predictive of better distance and line judgments (less error). Vine and Wilson (2010) did find significant differences between QE-trained and Control groups in their study, however this used novice performers and was carried out over a longer period (400 training putts). The lack of statistical difference in the current study is therefore likely due to a ceiling effect resulting from the use of elite golfers; within group variations in performance; and a reduction of statistical power caused by the loss of gaze data for some QE-trained participants.

\section{HYPOTHESIS 2: PRESSURE}

The purpose of the pressure test was to examine if QE training might offer further benefits in terms of helping golfers maintain effective QE durations even when under pressure (see Vine and Wilson, 2010). The MRF-3 data supports the effectiveness of the anxiety manipulation, indicating that both groups experienced significantly more cognitive anxiety during the pressure test than the retention test (Table 1). The anxiety levels are similar to those reported in previous research in both laboratory and competitive settings (e.g., Krane, 1994; Smith et al., 2001; Wilson et al., 2007; Vine and Wilson, 2010).

Consistent with the predictions of hypothesis 2, while both groups experienced comparable levels of anxiety, this had differential effects on their gaze control and performance. Specifically, while there were non-significant differences in $\mathrm{QE}$ at retention, the Control group displayed significantly shorter QE durations $($ mean $=1404 \mathrm{~ms})$ than the QE-trained group (mean = $2794 \mathrm{~ms}$; Figure 1) under pressure. This reduction in the efficiency of goaldirected control when anxious is consistent with the predictions of attentional control theory (Eysenck et al., 2007) which has previously been tested using the QE in basketball free-throw shooting (Wilson et al., 2009) and soccer penalty shooting (Wood and Wilson, 2010). Eysenck et al. (2007) argue that anxious individuals are more distractible, as anxiety increases the influence of the stimulus-driven attentional system at the expense of goal-directed control.

The impairment of effective attentional control (QE) experienced by the Control group resulted in subsequent performance degradation; or choking (Gucciardi et al., 2010). Specifically, the Control group only holed an average of $36 \%$ of putts during the pressure test (compared to $45 \%$ at retention), while the QE-trained group holed an average of $60 \%$ (compared to $59 \%$ at retention; Figure 1). Moreover, the Control group also exhibited significantly greater performance error than the QE-trained group during the pressure test (Figure 2). Thus, consistent with the study by Vickers and Williams (2007) individuals who maintained or increased their QE periods under pressure, were able to maintain or exceed performance from non-pressure performance levels. More importantly, the results mirror recent findings from a similar training study with novice golfers, revealing that performers can be trained to maintain effective QE periods (Vine and Wilson, 2010). In that study, the Control group also performed significantly worse in the pressure test than the QE-trained group, holing significantly fewer putts and missing by a greater distance.

\section{HYPOTHESIS 3: COMPETITIVE PERFORMANCE}

There were no differences in the competitive performance levels (putts per round or percentage of 6-10 ft putts holed) between both groups at baseline (pre-training), indicating that both groups started from similar levels of putting performance. Accordant with the predictions of hypothesis 3, the QE-trained group made 


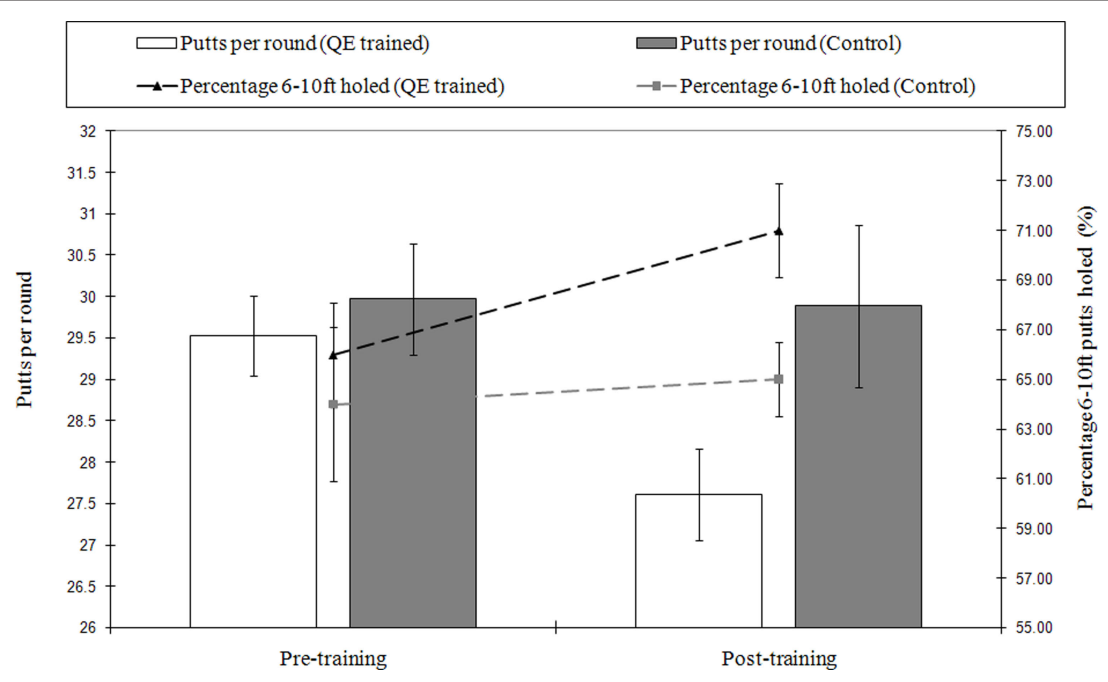

FIGURE 3 | Competitive performance: Number of putts per round and percentage 6-10 ft putts holed (\%) for QE-trained and Control groups during pre-training and post-training rounds (ISEM).

significantly fewer putts per round than the Control group at posttraining (27.6 vs. 29.9; Figure 3), demonstrating a positive transfer of training to competitive play (see Adolphe et al., 1997; Harle and Vickers, 2001). Specifically, the performance data revealed that while the Control group displayed no change in performance following training, the $\mathrm{QE}$-trained group reduced their mean number of putts per round by 1.92 shots and holed $5 \%$ more putts from 6 to $10 \mathrm{ft}$ (Figure 3).

\section{HOW DOES THE OE IMPROVE PERFORMANCE?}

There may be a number of mechanisms through which the QE impacts upon putting performance, consisting of both visuomotor control and psychological control elements. First, as highlighted for simple reaching and grasping tasks (e.g., Neggers and Bekkering, 2000); well-learned visually guided tasks (Land, 2009); and sport skills (Vickers, 2009), the motor system tends to be more accurate when provided with timely information about targets from the gaze system. In effect, by holding a ball focused QE throughout the putting stroke and through impact, golfers are able to ensure a more accurate contact with the sweet spot of the putter, ensuring more consistent ball strike. Second, information about the location of the hole may be more effectively stored in a visual memory buffer by maintaining a steady gaze on the ball, as this strategy reduces potential distractions from other visual sources and therefore allows this information to guide accurate putting actions (cf. Land and Furneaux, 1997; Wood and Wilson, 2010). Moreover, the movement parameters (e.g., direction, distance, and force), as well as the timing and co-ordination of the arms are fine-tuned in this crucial period of cognitive pre-programming leading to a more effective putting technique and successful performance (Vickers, 1992).

Third, the QE may provide the "external focus of attention" described by Wulf (2007) or the "external cue" described in Singer's (2002) five-step pre-performance routine. Singer advocates focusing on an external cue to prevent athletes from focusing on internal or external distracters, negative thoughts, or the mechanics of skill execution (Singer, 2002). As the stimulus-driven attentional system is more active when performers are anxious (Eysenck et al., 2007), such internal and external distracters are more likely to influence pressure putts. The QE may therefore also help provide a focus on what is controllable (ball strike) rather than what is not (a successful outcome) when golfers are under pressure. Fourth, the QE may simply help produce a general quiescence of the psychomotor system. Previous research has demonstrated that superior visuomotor performance is reflected in increased psychomotor and neural efficiency (Milton et al., 2007; Janelle and Hatfield, 2008; Yarrow et al., 2009). Subsequent research should therefore attempt to untangle how the QE exerts its influence on performance through these various pathways. The development of our understanding of these mechanisms may have implications for research in general attention and vision science, as well as in human movement science.

A final explanation for the positive results of $\mathrm{QE}$ training in the current study is that a Hawthorne effect may have been evident for the QE-trained group. By informing these participants that they were receiving instructions reflective of expert performance, they may have simply become more confident and motivated. While the Control group did receive almost the same training intervention (gaze feedback in relation to an expert model), they were not explicitly informed of the benefits of this technique. However, the regression analysis suggests that the $\mathrm{QE}$ itself strongly predicts variance in performance (43\%) across both groups during the lab-based retention and pressure tests. This finding suggests that the benefit of maintaining a longer QE period must be above and beyond any placebo effect due to being in the intervention group. However, as such motivational confounds cannot be fully refuted using the current design, future research should train a third group who should be taught a "placebo" intervention designed to control for motivational confounds.

\section{IMPLICATIONS}

The current study meets the call of Williams and Grant (1999) for research to attempt to determine whether pre-post differences in clinical settings transfer to the sporting domain. In this sense the 
current study furthers the work of Vine and Wilson (2010) and shows that the benefits of QE training may transfer to the real competitive world of golf, and to experienced golfers as well as novices. The findings of the present study suggest that the QE strategy is easily incorporated as part of a pre-performance routine that can be learned quickly and applied on the course. Pre-performance routines (task relevant behavioral and cognitive routines) have long been posited as a useful strategy for maintaining effective concentration in pressurized environments (e.g., Moran, 1996) and for alleviating choking (Mesagno et al., 2008). Indeed golfers themselves have identified attentional control; the ability to maintain concentration and resist distraction, as a critical psychological skill for optimal performance (Bois et al., 2009; Hellstrom, 2009b).

It has been highlighted that at high levels of golf, even minor improvements in performance can make a major difference (Cohn et al., 1990). When compared to putting statistics from the current PGA Tour season the magnitude of the recorded improvements for these golfers are substantiated. For example, according to the 2010 PGA Tour putts per round statistics, if a golfer ranked last (195th), experienced an improvement of 1.92 putts per round, he would climb 167 places to 28th in the rankings for this statistic (PGA Tour Putting Statistics, 2010). Furthermore, as research has shown that a low number of putts per round is strongly associated with better overall golf performance (lower scores) and higher earnings on the PGA Tour (see Hellstrom, 2009a for a review), this improvement is likely to be highly beneficial to a golfer. However, it should be noted that the putts per round measure of performance may have been influenced by the participants' short game performance (i.e., chip, pitch, or bunker shots) and should be acknowledged as a limitation. Therefore, future research should perhaps adopt the putts per greens reached in regulation [GIR; the number of greens reached in two shots (or less) than par for the hole] as a more sensitive measure of competitive putting performance (see Hellstrom, 2009a).

The findings of this study also have implications for other environments where visuomotor skills need to be performed under pressure. For example, Wilson and colleagues have recently

\section{REFERENCES}

Adolphe, R.M., Vickers, J.N., and LaPlante, G. (1997). The effects of training visual attention on gaze behaviour and accuracy: a pilot study. Int. J. Sports Vision 4, 28-33.

Behan, M., and Wilson, M. (2008). State anxiety and visual attention: the role of the quiet eye period in aiming to a far target. J. Sports Sci. 26, 207-215.

Bois, J. E., Sarrazin, P. G., Southon, J., and Boiche, J. C. S. (2009). Psychological characteristics and their relation to performance in professional golfers. Sport Psychol. 23, 252-270.

Cohn, P. J., Rotella, R. J., and Lloyd, J. W. (1990). Effects of a cognitive-behavioral intervention on the preshot routine and performance in golf. Sport Psychol. 4, 33-47.

Cooke, A., Kavussanu, M., McIntyre, D., and Ring, C. (2010). Psychological, muscular and kinematic factors

examined differences in the gaze behavior of experienced and novice laparoscopic surgeons performing training tasks in virtual reality (Wilson et al., 2010a; Wilson et al., in press). The authors suggest that surgeons (like golfers) may benefit from training instructions that guide them to fixate on key targets in order to help guide motor control (Wilson et al., 2010b). The benefits of gaze training in improving motor performance have also been demonstrated in environments where individuals find it difficult to make accurate eye movements (e.g., cerebellar patients; Crowdy et al., 2002), or where motor performance is impaired (e.g., elderly stair climbers; Young and Hollands, 2010). Explicit QE training instructions may be a useful addition to these interventions, especially due to the predicted benefits for both visuomotor and psychological control.

\section{CONCLUSION}

To conclude, the current study investigated the effectiveness of a QE training intervention aimed at optimizing the gaze control and performance of elite golfers in both a laboratory-based putting task and real competitive performance. Consistent with previous research and predictions, the $\mathrm{QE}$ intervention acted to insulate golfers from the debilitating effects of anxiety upon performance by ensuring they maintained effective gaze control (QE durations) in a pressurized environment. Moreover, the QE-trained group reported significantly improved performance in a competitive setting, with an improvement in competitive putting performance of 1.92 putts per round and $5 \%$ more holed putts from 6 to $10 \mathrm{ft}$. From an applied perspective, the findings suggest that $\mathrm{QE}$ training may provide a useful psychological technique, as part of a pre-performance routine, to aid performance under pressure and improve performance in competitive environments.

\section{ACKNOWLEDGMENT}

This research was funded by a business voucher from the Economic and Social Research Council's Capacity Building Cluster in Sport, Leisure and Tourism, based at the University of Exeter (RES-187-24-0002).

tives on choking under pressure. J. Sport Exerc. Psychol. 32, 61-83.

Harle, S. K., and Vickers, J. N. (2001). Training quiet eye improves accuracy in the basketball free throw. Sport Psychol. 15, 289-305.

Hellstrom, J. (2009a). Competitive elite golf: a review of the relationships between playing results, technique and physique. Sports Med. 39, 723-741.

Hellstrom, J. (2009b). Psychological hallmarks of skilled golfers. Sports Med. 39, 845-855.

Janelle, C. M. (2002). Anxiety, arousal and visual attention: a mechanistic account of performance variability. J. Sports Sci. 20, 237-251.

Janelle, C. M., and Hatfield, B. (2008). Visual attention and brain processes that underlie expert performance: implications for sport and military psychology. Mil. Psychol. 20, 117-134.
Karlsen, J., Smith, G., and Nilsson, J. (2008). The stroke has only a minor influence on direction consistency in golf putting among elite players. $J$. Sports Sci. 26, 243-250.

Krane, V. (1994). The mental readiness form as a measure of competitive state anxiety. Sport Psychol. 8, 189-202.

Land, M. F. (2009). Vision, eye movements, and natural behavior. Vis. Neurosci. 26, 51-62.

Land, M. F., and Furneaux, S. (1997). The knowledge base of the oculomotor system. Philos. Trans. R. Soc. Lond., B, Biol. Sci. 352, 1231-1239.

Mann, D. T. Y., Williams, A. M., Ward, P., and Janelle, C. M. (2007). Perceptualcognitive expertise in sport: a metaanalysis. J. Sport Exerc. Psychol. 29, 457-478.

Martens, R., Burton, D., Vealey, R., Bump, L., and Smith,D. (1990). "Development of the CSAI-2," in Competitive Anxiety 
in Sport, eds R. Martens, R. Vealey, and D. Burton (Champaign IL: Human Kinetics), 127-140.

Mesagno, C., Marchant, D., and Morris, T. (2008). A pre-performance routine to alleviate choking in "chokingsusceptible" athletes. Sport Psychol. $22,439-459$.

Milton, J., Solodkin, A., Hlustik, P., and Small, S. L. (2007). The mind of expert motor performance is cool and focused. Neuroimage 35, 804-813.

Moran, A. P. (1996). The Psychology of Concentration in Sports Performers: A Cognitive Analysis. Hove: Psychology Press.

Neggers, S. F.W., and Bekkering, H. (2000). Ocular gaze is anchored to the target of an ongoing pointing movement. J. Neurophysiol. 83, 639-651.

Pelz, D. (2000). Dave Pelz's Putting Bible. New York: Doubleday.

PGA Tour Putting Statistics. (2010). Accessed May 25, 2010. Available at: http://www.pgatour.com/r/stats/ info/?343.

Singer, R. N. (2002). Preperformance state, routines and automaticity: what does it take to realise expertise in self paced events? J. Sport Exerc. Psychol. 24, 359-375.

Smith, N.C., Bellamy,M.,Collins, D.J., and Newell, D. (2001). A test of processing efficiency theory in a team sport context. J. Sport Sci. 19, 321-332.

Tierney, D. E., and Coop, R. H. (1998). "A bivariate probability model for putting proficiency," in Science and
Golf III: Proceedings of the 1998 World Scientific Congress of Golf, eds. M. R. Farally and A. J.Cochran (Champaign, IL: Human Kinetics), 385-394.

Vickers, J. N. (1992). Gaze control in golf putting. Perception 21, 117-132.

Vickers, J. N. (1996). Visual control when aiming at a far target. J. Exp. Psychol. Hum. Percept. Perform. 22, 342-354.

Vickers, J. N. (2007). Perception, cognition and decision training: the quiet eye in action. Champaign, IL: Human Kinetics.

Vickers, J.N. (2009). Advances in coupling perception and action: the quiet eye as a bidirectional link between gaze, attention, and action. Prog. Brain Res. 174, 279-288.

Vickers, J. N., and Williams, A. M. (2007). Performing under pressure: the effects of physiological arousal, cognitive anxiety, and gaze control in biathlon. J. Mot. Behav. 39, 381-394.

Vine, S. J., and Wilson, M. R. (2010). Quiet eye training helps to maintain effective attentional control and performance under pressure. J. Appl. Sport Psychol. 22, 361-376.

Williams, A. M., and Grant, A. (1999). Training perceptual skill in sport. Int. J. Sport Psychol. 30, 194-220.

Wilson, M. (2008). From processing efficiency to attentional control: a mechanistic account of the anxietyperformance relationship. Int. Rev. Sport Exerc. Psychol. 1, 184-201.

Wilson, M., McGrath, J., and Coleman, M. (2010b). Developing basic eye-hand coordination skills for laparoscopic surgery using gaze training. BJU Int. 105, 1356-1358

Wilson, M., Smith, N.C., and Holmes, P.S (2007).The role of effort in influencing the effect of anxiety on performance: testing the conflicting predictions of processing efficiency theory and the conscious processing hypothesis. $\mathrm{Br}$. J. Psychol. 98, 411-428.

Wilson, M. R., McGrath, J., Vine, S. J., Brewer, J., Defriend, D., and Masters, R. S. W. (2010a). Psychomotor control in a virtual laparoscopic surgery training environment: gaze control parameters differentiate novices from experts. Surg. Endosc. 24, 2458-2464.

Wilson, M. R., McGrath, J., Vine, S. J., Brewer, J., Defriend, D., and Masters R. S. W. (in press). Perceptual impairment and visuomotor control in virtual laparoscopic surgery. Surg. Endosc.

Wilson, M. R., and Pearcey, R. (2009). On the right line: the visuomotor control of straight and breaking golf putts. Percept. Mot. Skills 109, 555-562.

Wilson, M. R., Vine, S. J., and Wood, G. (2009). The influence of anxiety on visual attentional control in basketball free throw shooting. J. Sport Exerc. Psychol. 31, 1-19.

Wood, G., and Wilson, M. R. (2010) Goalkeeper distractions increase the likelihood of missing a penalty kick. J. Sports Sci. 28, 937-946.

Wulf, G. (2007).Attention and Motor Skill Learning. Champaign, IL: Human Kinematics.
Yarrow, K., Brown, P., and Krakauer, J. W. (2009). Inside the brain of an elite athlete: the neural processes that support high achievement in sports. Nat. Rev Neurosci. 10, 585-596.

Young, W. R., and Hollands, M.A. (2010). Can telling older adults where to look reduce falls? Evidence for a causal link between inappropriate visual sampling and suboptimal stepping performance. Exp. Brain Res. 204, 103-113.

Conflict of Interest Statement: The authors declare that the research was conducted in the absence of any commercial or financial relationships that could be construed as a potential conflict of interest.

Received: 14 September 2010; accepted: 06 January 2011; published online: 28 January 2011.

Citation: Vine SJ, Moore LJ and Wilson MR (2011) Quiet eye training facilitates competitive putting performance in elite golfers. Front. Psychology 2:8. doi: 10.3389/ fpsyg.2011.00008

This article was submitted to Frontiers in Movement Science and Sport Psychology, a specialty of Frontiers in Psychology. Copyright (c) 2011 Vine, Moore and Wilson. This is an open-access article subject to an exclusive license agreement between the authors and Frontiers Media $S A$, which permits unrestricted use, distribution, and reproduction in any medium, provided the original authors and source are credited. 